\title{
Group Decision Making with Incomplete Information: a dominance and quasi-optimality volume-based approach using Monte-Carlo simulation
}

\author{
February 18, 2016
}

\begin{abstract}
In this paper, we present a comprehensive framework for multi-attribute group decision making considering that neither information about individual preferences nor the importance of individual decision makers for the group is available in exact form. We study several different forms of incomplete preference information, including a ranking of attribute weights, a ranking of values of alternatives in each attribute, and a ranking of value differences. Our approach is based on relative volumes in parameter space and allows for probabilistic statements about different results, including optimality or quasi-optimality of alternatives, or relations between alternatives.
\end{abstract}

Keywords: Multiple criteria analysis, group decisions, incomplete information, ordinal information, additive model.

\section{Introduction}

Complex and important decision problems in organizations often require the consideration of multiple attributes, and are frequently made by groups of experts or decision makers, who are able to analyze the problem from different perspectives. Over the last decades, multi-attribute group decisions have thus evolved into a major area of interest in decision analysis (Hwang and Lim, 1987; Belton and Stewart, 2002). Despite several decades of research, there are still many challenges to the effective support of decision makers in a multi-attribute group decision context. Perhaps the most critical issue in this context is the amount and type of information that group members need to provide in order to reach a group decision. Even single decision makers, who do not operate in a group context, sometimes have difficulties in specifying the preference information needed to solve a multi-criteria decision problem exactly (Damart et al., 2007). This problem is even more severe in a group context, where aggregation of opinions across group members might require additional information, e.g. about the importance of each member's opinion for the group decision.

Existing literature on group decision analysis (for multi-attribute problems and in more general settings) thus employs a wide spectrum of different types of information to be provided by group members. One end of this spectrum is formed by models of social choice theory, which only require group members to specify an ordinal ranking of alternatives and thus pose very weak requirements on their ability to provide information. The downside of this approach is the well known impossibility theorem by Arrow (1951), which indicates that it is not possible to find an aggregation of rankings that produces a transitive and complete group ranking and simultaneously fulfills four reasonable conditions (universal domain, Pareto optimality, independence of irrelevant alternatives and non-dictatorship).

At the other end of the spectrum, many approaches to multi-attribute group decision making require group members to specify a complete multi-attribute value function (Watson and Buede, 1987) or multiattribute utility function (Keeney and Raiffa, 1976) (the difference between these two functions is not relevant for our work), and then combine these individual functions in a group utility function (Dyer and Sarin, 1979; Keeney and Kirkwood, 1975; Keeney and Nau, 2011; Keeney, 2013). It can be shown (Keeney, 1976) that a group utility function, which additively combines individual utility functions weighted by weights of each decision maker, fulfills axioms analogous to those of Arrow (1951). However, specification 
of these individual utility functions is already a complex task for the group members, and the additional specification of member weights further adds to this complexity.

The approach which we pursue in this paper attempts to cover a middle ground between these two ends of the spectrum by requiring less information from group members than the complete specification of cardinal utilities, and at the same time avoiding (as far as possible) violation of Arrow's axioms. Our approach builds upon models of decision making under incomplete (partial, imprecise) information (Dias and Clímaco, 2000; Salo and Hämäläinen, 2001; Weber, 1987). These methods allow Decision Makers (DMs) to specify information on preference parameters only imprecisely, for example in the form of linear constraints that can be defined directly (Hazen, 1986; Kirkwood and Sarin, 1985; Park and Kim, 1997) or indirectly via a comparison of alternatives (Greco et al., 2008), rather than as exact values. In particular, our approach is similar to Stochastic Multicriteria Acceptability Analysis (SMAA) (Lahdelma et al., 1998) which utilizes incomplete preference information to provide probabilistic statements about possible relations between alternatives as well as their ranks.

Methods for decision making under incomplete information have been proposed as extensions to many different methods for multi-criteria decision making. The original SMAA method (Lahdelma et al., 1998) builds upon additive multi-attribute utility functions, and it has been extended later to many other multi-criteria decision making methods (Tervonen and Figueira, 2008). Likewise, different methods to aggregate individual evaluations based on various multi-criteria methods to a group evaluation exist. Such aggregation can take place at the level of preference parameters, or at the level of evaluations of alternatives (Vetschera, 1990). In the first case, a multi-criteria method is applied at the group level using aggregated parameters elicited from the entire group, or computed from individual parameters. In the second case, group members first evaluate alternatives individually using some multi-attribute method, and then the evaluations are aggregated across members. Both approaches could be applied to different multi-criteria methods, leading to a variety of multi-attribute group decision methods. In view of the already mentioned axiomatic results, which show that additive multi-attribute utility functions can consistently be aggregated to a group utility function, the present paper uses multi-attribute utility functions as the individual preference model.

In the context of additive aggregation of individual additive multi-attribute utility functions, complete information would require the specification of three types of preference parameters: (i) The individual values that group members assign to each possible value in each attribute, (ii) the weights that each DM attaches to each partial value function (the attribute weights), and (iii) the weights attached to the utilities of each group member (the DMs' weights). In the present paper, we consider different levels of incomplete information on each of these parameters. For values assigned to the outcomes in each attribute, we consider three possibilities: that these values are known exactly; that only a ranking of outcomes (but not the exact values assigned to them) is known; and as an intermediate level of information, that a ranking of values, as well as a ranking of adjacent value differences is known. This last case refers to a situation in which the decision maker is able to indicate (in an ordinal way) the strength of preference between pairs of consecutive outcomes in an attribute. For attribute weights, we consider the case that weights are known exactly, and that a ranking of weights is known (i.e. that the decision maker is able to indicate a ranking of the "importance" of attributes). Since weights of decision makers in the aggregate group utility are very difficult to determine, we do not consider a situation in which these weights are exactly known. We distinguish between two cases for these parameters: one in which weights are completely unknown, and one case in which decision maker weights are restricted so that no group member receives $50 \%$ or more of the total weight. The latter prevents decision makers from becoming a "strategic dictator" as defined by Dias and Sarabando (2012).

The methodology we propose exploits this incomplete information in a comprehensive way. It aims at identifying optimal or quasi-optimal alternatives for the group (which will be defined subsequently), and to establish different types of relations between alternatives. To provide even richer information, we propose to base the analysis on the relative volumes of the parameter space that lead to the conclusions of interest. Under the assumption of a uniform distribution of parameters, this allows us to make probability statements about the properties of alternatives (such as preference or optimality) analogously to the existing body of literature on using domains, e.g., Bana e Costa (1986), Charnetski and Soland (1978), or Lahdelma and Salminen (2001).

In the next section we briefly review some approaches related to the use of incomplete information in group decision making problems. In Section 3, we introduce our approach. Section 4 presents an 
illustrative example. We finish in Section 5 with some conclusions and some lines for future research.

\section{Group decision making with incomplete information: a brief review}

Several approaches for group decision making with incomplete information have been developed in literature. In this review, we consider methods in which the incomplete information is provided by means of defining constraints on possible parameter values, often reflecting a ranking of alternatives and thus ordinal information.

Following similar methods for individual decision making, most early methods use mathematical programming to establish dominance between alternatives (Weber, 1987; Hazen, 1986). Dominance here refers to the set of possible parameter vectors and an alternative is said to dominate another one, if it is better for all possible parameter vectors. However, dominance is often hard to establish, so other methods aim at providing additional information beyond this dominance relation. One can reduce the set of feasible parameters to one "representative" parameter vector (Kadzinski et al., 2012) and thus obtain a unique group utility function. Other methods consider the entire set of feasible parameters, and for example use volumes to make probabilistic statements about preferences (Sarabando et al., 2013). Sarabando et al. (2013) propose to derive incomplete information about the preferences of negotiators from the statements they make and the offers they exchange during the negotiation process. They present and discuss three approaches that use this information in order to help a mediator proposing a better solution than the compromise the negotiators have reached or are close to reach. The third approach uses a domain-based analysis and calculates the relative volume of the feasible set of parameter values for which some conditions are fulfilled.

There are several methods that use mathematical programming to derive dominance relations. As an example, Salo (1995) proposes an interactive method based on an additive value function with imprecise inputs. The method alternates between phases of preference elicitation and the computation of intermediate results. As the DMs indicate their preferences in more detail, the set of non-dominated alternatives gradually becomes smaller. Kim and Ahn (1999) extend standard optimization-based models of decision making under incomplete information to the group context using the concept of dominance. This model is rather general and allows the DM's weights, attribute weights, and values to be incompletely specified.

Some of the earliest methods to deal with incomplete information are based on the idea of volume computations, e.g., Bana e Costa (1986) and Charnetski and Soland (1978). More recently, the SMAA methods (Lahdelma et al., 1998; Lahdelma and Salminen, 2001) emphasize the exploration of parameter space. Instead of trying to identify the best alternative based on elicited information, these methods identify parameter settings in which one alternative is preferred to another one. SMAA uses simulation methods to compute for example the probabilities that an alternative occupies each position in the ranking of all alternatives. Since SMAA uses simulation, it allows for very general inputs on a DM's preferences, while optimization-based methods are restricted to statements that can be represented as constraints in a (linear) optimization problem. The method proposed by Vetschera (2008) to measure the extent to which information about the preferences is available during a negotiation is also based on the domain criterion, but uses an exact algorithm to calculate volumes and is therefore also restricted to linear constraints.

Jiménez, Mateos, and collaborators use simulation in order to identify a consensus strategy or to aggregate the preferences of DMs. Mateos et al. (2003) develop a decision support system based on Monte-Carlo simulation techniques, where the DMs' preferences are elicited separately, as a starting point for a negotiation process. In later work (Jiménez et al., 2005), they propose an iterative process to achieve a consensus strategy. In each iteration, the imprecise sets of utilities and weights corresponding to the different DMs are successively tightened.

Distance-based models were suggested by Kemeny and Snell (1962), who proposed to measure the degree of correlation among rankings. There are other methods that focus on the calculation of distances: to aggregate sets of binary relations (Cook and Kress, 1985; Jabeur and Martel, 2207; Khélifa and Martel, 2001), to aggregate a vector of weights (Contreras and Marmol, 2007), or to implement an interactive approach (Eklund et al., 2007). Tavares (2004) deals with the aggregation of rankings in a problem about distances between attribute weights. 
Finally, there exist methods (e.g., by Dias and Clímaco (2005) and Hodgkin et al. (2005)) intended to provide feedback information to the DMs, instead of simplifying the information by aggregation.

\section{Volume and Dominance Approach}

\subsection{Introduction}

We assume that a group consisting of $k \mathrm{DMs}(k \geq 2)$ has to select one best alternative out of a set $A$ of $m$ alternatives. The DMs consider $n$ attributes in their evaluation of alternatives, and their individual preferences can be represented by additive multi-attribute value functions (Keeney and Raiffa, 1976). Additive preference models are recommended in the context of group decision making problems, according to Raiffa (1982), because of their robustness, transparency, and ease of development.

We furthermore assume, in accordance with Keeney and Kirkwood (1975), Dyer and Sarin (1979), and others, that aggregation across DMs is performed by an additive group utility function. Therefore, the additive value representation of an alternative $x^{(p)} \in A$ can be written as:

$$
v\left(x^{(p)}\right)=\sum_{i=1}^{k} d_{i} \sum_{j=1}^{n} w_{i j} v_{i j}\left(x_{j}^{(p)}\right)
$$

where $d_{i}$ is the importance weight of DM $i, w_{i j}$ is the weight of attribute $j$ for DM $i, v_{i j}($.$) is the value$ function of DM $i$ on attribute $j$. For a given and finite set of alternatives, there is only a finite set of values each attribute can take on. To simplify the notation, we therefore denote the single-attribute utility which DM $i$ assigns to the outcome of alternative $x^{(p)}$ in attribute $j$ by $s_{i j}^{p}$ and the global (multiattribute) utility which DM $i$ assigns to the outcome vector of alternative $x^{(p)}$ by $g_{i}^{p}$. We thus can therefore rewrite (3.1) as:

$$
v\left(x^{(p)}\right)=\sum_{i=1}^{k} d_{i} \sum_{j=1}^{n} w_{i j} v_{i j}\left(x_{j}^{(p)}\right)=\sum_{i=1}^{k} d_{i} \sum_{j=1}^{n} w_{i j} s_{i j}^{p}=\sum_{i=1}^{k} d_{i} g_{i}^{p} .
$$

According to Eq. (3.1), all DMs evaluate the same set of alternatives according to the same set of attributes. In practice, different DMs might use different attributes in their evaluation of alternatives. For example, in selecting different types of equipment to purchase, representatives of the finance department might use financial attributes, while representatives of the production department might use technical attributes. This situation can formally be represented in (3.1) by considering the union of the attribute sets of all DMs and setting the weight $w_{i j}$ to zero if DM $i$ is not interested in attribute $j$.

Attribute weights indicate the relative importance of changing the level of performance on the respective attribute from its worst level (corresponding to a value of zero) to the best level (which is represented by a value of one). Many methods have been proposed in literature to provide exact values of attribute weights, e.g. the pricing-out (Keeney and Raiffa, 1976) and the Swing (Winterfeldt and Edwards, 1986) methods. The single attribute values for the alternatives can be determined through direct rating or through indifference techniques (as the bisection method) (Winterfeldt and Edwards, 1986). However, empirical evidence suggests that different methods lead to different results (Schoemaker and Waid, 1982; Pöyhönen and Hämäläinen, 2001), and consequently "true" weights or values are more or less impossible to obtain. For this reason, we consider methods of decision making under incomplete information, which do not require exact specification of parameters.

The assessment of the DMs' importance weights, $d_{i}$, is another difficulty associated with the use of the additive value function (3.1). The choice of importance weights requires comparisons concerning the authority, experience and expertise of the DMs. Salo (1995) proposes to calculate importance weights by taking the convex hull of all weights which the DMs would assign to each other. Furthermore, DMs could establish reasonable restrictions on their importance weights through an open discussion, or by asking for comparisons from possibly dissenting coalitions. Sen (1970) suggests not to specify exact weights, but to work with a reasonable range of importance weights (even if the results from the imprecise model may be incomplete).

In this work, we follow the arguments of Sen and present an approach which avoids the elicitation of DMs' importance weights. This should benefit the alternatives that are potentially good compromises for 
a large majority. We also assume that the information about the attribute weights and about the value functions is incomplete, but constrained by elicited information. We consider two cases: That values are known exactly and only attribute weights are uncertain, and that both attribute weights and values are uncertain.

\subsection{Characterization of the information about attribute weights and values}

The assessment of the attribute weights is often considered to be a cognitively demanding task (Alfares and Duffuaa, 2008) and, in some circumstances, it may be appropriate to ask DMs to provide only ordinal information about the attribute weights, rather than a direct assessment of their numerical values. Let us consider, without loss of generality, that the attribute indexes are coded such that the weights are in decreasing order according to ordinal information provided by the DMs. This information could be obtained, for example, using swings (Winterfeldt and Edwards, 1986; Edwards and Barron, 1994) between the worst and best values in each attribute. For DM $i(i=1, \ldots, k)$, the set of weight vectors compatible with this ordinal information is:

$$
W_{i}=\left\{\left(w_{i 1}, w_{i 2}, \ldots, w_{i n}\right): w_{i 1} \geq w_{i 2} \geq \ldots \geq w_{i n} \geq 0, \sum_{t=1}^{n} w_{i t}=1\right\},
$$

where $w_{i t} \in\left\{w_{i 1}, \ldots, w_{i n}\right\}$ represents the weight in the position $t$ for DM $i$, for $t=1, \ldots, n$. We follow the convention that weights are non-negative and they add up to 1 .

We also assume that the information related to the value of each alternative in each attribute is incomplete. We first consider the case that each DM provides ordinal information about the value of each alternative in each attribute. Denote by $x_{j}^{l}$ the $l$-th possible outcome in attribute $j$ and by $s_{i j}^{l}$ the partial utility assigned by DM $i$ to that outcome. Furthermore we assume, without loss of generality, that outcomes are numbered according to the preferences of that DM in decreasing order so that the DM prefers $x_{j}^{l}$ over $x_{j}^{l+1}$. Given the DM's ranking of $m_{j}$ different outcomes, we can define the following constraint on the values $s_{i j}^{l}$ :

$$
V_{i j}=\left\{\left(s_{i j}^{1}, s_{i j}^{2}, \ldots, s_{i j}^{m_{j}}\right): 1=s_{i j}^{1} \geq s_{i j}^{2} \geq \ldots \geq s_{i j}^{m_{j}}=0\right\}
$$

Note that this does not imply that all decision makers rank outcomes in the same way, since this calculation is performed separately for each DM.

Considering all attributes, we can define

$$
V_{i}=V_{i 1} \times V_{i 2} \times \ldots \times V_{i n}
$$

as the set of possible value vectors that is compatible with DM $i$ 's rankings of outcomes.

Alternatively, we also consider the possibility that each DM provides ordinal information about the difference of values between two consecutive outcomes in each attribute. We define $\Delta_{i j}^{l}=s_{i j}^{l}-s_{i j}^{l+1} \geq 0$. Furthermore, assume that the DM indicates that he or she considers $\Delta_{i j}^{[1]}$ to be the largest difference, $\Delta_{i j}^{[2]}$ the second largest and so on, which leads to the constraints:

$$
\Delta_{i j}^{[1]} \geq \Delta_{i j}^{[2]} \geq \ldots \Delta_{i j}^{\left[m_{j}-1\right]}
$$

\subsection{Characterization of the DMs' weights}

Let $D$ denote the set of accepted weight vectors for the DMs. We consider two methods to avoid that the group has to explicitly elicit importance weights. First, we assume that weights are completely free to vary:

$$
D=\left\{\left(d_{1}, d_{2}, \ldots, d_{k}\right): d_{1}, d_{2}, \ldots, d_{k} \geq 0, \sum_{j=1}^{k} d_{j}=1\right\}
$$


However, this would allow any DM to receive such a large weight that this DM could determine the group ranking and thus could be considered a "dictator". According to Arrow (1951), a DM $y$ is a dictator if $x^{(i)} \succ_{y} x^{(p)} \Rightarrow x^{(i)} \succ x^{(p)}$. In other words, DM $y$ is a dictator if it is sufficient that DM $y$ says that $g_{y}^{i}>g_{y}^{p}$, independently of the values that the other DMs give to the two alternatives, in order to establish the group preference $x^{(i)} \succ x^{(p)}$. Note that by Arrow's definition, the group would choose alternative $x^{(i)}$, even if, for the dictator, the intensity of preference of $x^{(i)}$ over $x^{(p)}$ is small. Most of the literature characterizing group utility functions (Dyer and Sarin, 1979; Keeney and Kirkwood, 1975; Keeney, 1976) also considers a DM to be a dictator if that person's preferences determine group preference regardless of the preferences of the other DMs. The axiomatization of Keeney (1976) considers that it is sufficient that weights comply with (3.7), i.e, are strictly positive, in order to ensure nondictatorship in Arrow's sense.

The conditions stated in (3.7), however, might not correspond to an intuitive notion of a dictator. For instance, in a case with $3 \mathrm{DMs}$, one can argue that $d=(0.98,0.01,0.01)$ should not be an admissible weight vector, since the first DM is much more important than the other two DMs. Indeed, if there are 5 alternatives $x^{(1)}, x^{(2)}, x^{(3)}, x^{(4)}$, and $x^{(5)}$, and individual 1 declares for instance $g_{1}^{1}=1, g_{1}^{2}=0.75, g_{1}^{3}=0.5$, $g_{1}^{4}=0.25$, and $g_{1}^{5}=0$, then he would impose the ranking $x^{(1)} \succ x^{(2)} \succ x^{(3)} \succ x^{(4)} \succ x^{(5)}$ even if this totally contradicts the utilities of individuals 2 and 3.

A recent characterization of group utility functions (Dias and Sarabando, 2012) proposed to use an alternative definition of nondictatorship, suggesting (among other more constraining possibilities) a condition of "Immunity to Imposition of a Winner (IIW) by an individual": there is no individual with the property that he can indicate preferences in a way that guarantees that his preferred alternative has a group utility strictly greater than the utilities of all other alternatives, regardless of all other individuals' preferences. According to this characterization, which acknowledges the possibility of an individual misrepresenting his preferences to impose a winner, this would require that no individual has an importance weight higher than 0.5. As an alternative to (3.7) we will consider the following set of acceptable weights that prevent the existence of a "strategic dictator" in the sense of IIW:

$$
D=\left\{\left(d_{1}, d_{2}, \ldots, d_{k}\right): 0.5>d_{1}, d_{2}, \ldots, d_{k} \geq 0, \sum_{j=1}^{k} d_{j}=1\right\}
$$

\subsection{Methodology}

\subsubsection{General ideas}

As we have already mentioned in the preceding sections, previous approaches to (group) decision making under incomplete information can roughly be classified into two classes of methods: methods using mathematical programming formulations to establish dominance between alternatives or other properties of alternatives, and volume-based methods (using simulation or exact algorithms), which aim for probabilistic statements. Methods of the first group consider properties of alternatives (like optimality or efficiency) or relations between alternatives (namely the preference relation). Given that many parameter vectors might be compatible with the information provided by the DM, one aims to find properties which either hold for all parameter vectors, or which hold for a least one parameter vector. In the terminology introduced by Greco et al. (2008), the first type of properties is called necessary, and the second type possible. Necessary preference is also referred to as dominance.

Necessary and possible properties refer to extreme cases, in which a property holds for all, or perhaps just one, possible parameter vector. An axiomatic characterization of these relations has been developed by Giarlotta and Greco (2013). Volume-based methods aim to provide additional information for situations in between these extremes. They consider parameter vectors to be randomly drawn from the set of parameter vectors which are compatible with the preference information available, and compute the probability that a certain property will hold for a randomly selected parameter vector. Under the assumption that parameter vectors are uniformly distributed, this probability corresponds to the relative volume of the parameter space region compatible with available information where the property studied holds. The basic assumption behind this approach is that, if an alternative is possibly optimal, then DMs would like to know whether there is a $99 \%$ chance that a selected alternative will actually be optimal, or for instance, this chance is only $1 \%$. If the information available can be represented in the form of linear 
constraints on parameters, this region forms a polyhedron in multidimensional space and its volume can be computed by exact algorithms. However, for many applications, simulation-based methods are more appropriate, since they allow to analyze several properties simultaneously.

The approach followed in this paper combines elements of the volume computation approaches and the dominance-based approaches: it consists in exploring the parameter space defined by the constraints presented in the previous subsections, so that we can measure the relative volume of the feasible set of parameter values where certain properties hold. An analogous approach has been suggested for the context of bilateral negotiations with incomplete information (Sarabando et al., 2013).

Let us denote by $S$ the set of feasible parameter values given the information currently available:

$$
S=W_{1} \times V_{1} \times W_{2} \times V_{2} \times \ldots \times W_{k} \times V_{k} \times D
$$

where $W_{i}$ and $V_{i}$ are the feasible sets of attribute weights and values, respectively, for DM $i(i=1, \ldots, k)$, and $D$ the feasible set of DMs' weights.

Let $\operatorname{Vol}(S)$ denote the volume of the set $S$. To measure the relative volume of the parameter space where each alternative $x^{(p)}$ is the best one, we can determine $\operatorname{Vol}\left(T: v\left(x^{(p)}\right) \geq v\left(x^{(j)}\right), \forall j\right) / \operatorname{Vol}(S)$. It is also possible to consider quasi-optimality (Dias and Clímaco, 2000) instead of strict optimality, to highlight those alternatives that are always close to the best ones in terms of difference of value. For a given vector of parameters, quasi-optimal alternatives are either the best ones or the ones that do not lose to the best ones for a difference greater than a specified tolerance $\epsilon>0$. The quasi-optimality relative volume for an alternative $x^{(p)}$ is $\operatorname{Vol}\left(T: v\left(x^{(p)}\right) \geq v\left(x^{(j)}\right)-\epsilon, \forall j\right) / \operatorname{Vol}(S)$. This allows concluding, for instance, that "alternative $x^{(p)}$ is quasi-optimal allowing a tolerance of $\epsilon=0.01$, for $93 \%$ of the possible parameter values".

Since nonlinear relations hinder the computation of volumes by exact methods, Monte-Carlo simulation can be used to obtain good approximations, analogously to SMAA methods (Lahdelma and Salminen, 2001; Lahdelma et al., 2003). The SMAA methods calculate the acceptability of an alternative for each position in a ranking as volumes in the weights space. We generalize this approach and use simulation to simultaneously test for the following properties of alternatives:

- Optimality

- Quasi-optimality

- (Necessary) Preference between alternatives

While optimality volumes can be useful to highlight the most promising alternatives, the necessary preference relation can be used to discard poor alternatives. Alternative $x^{(j)}$ can be said to be necessarily preferred to (or "dominate") alternative $x^{(i)}$ if the value of $x^{(j)}$ is at least as good as the value of $x^{(i)}$, for all feasible parameter values (Kim and Ahn, 1999). This property could be tested using an optimization model. Since we already obtain the evaluations of all alternatives for all parameter vectors tested from the simulation, it can also directly be verified from the simulation results. It is also possible to check for potential preference, which occurs when an alternative is better than the other one for at least one of the possible parameter vectors. In general, we can compute a probabilistic preference relation since the relative volume of the parameter space in which one alternative is preferred to another one represents the probability of this preference. This concept is similar to the pairwise winning indices in SMAA (Leskinen et al., 2006; Kadzinski and Tervonen, 2013). Note that we use the term "necessary preference" synonymously with "dominance", which refers to the set of possible parameter vectors. Since we are interested in an aggregate group utility, we do not refer to Pareto optimality in the space of DM's utilities (i.e. one alternative dominates another one if it makes at least one DM better off and no one worse off). This type of dominance could, however, also be studied using our approach.

We suggest to randomly generate the values of the parameters, using a uniform distribution, and to use the following procedure:

i. Consider a given number of iterations $n_{i t r}$ and set counter cont $=1$;

ii. Generate $w_{i j}$, for $i=1, \ldots, k$ and $j=1, \ldots, n$ fulfilling the required constraints;

iii. Generate $s_{i j}^{p}$, for $i=1, \ldots, k, j=1, \ldots, n$ and $p=1, \ldots, m$ fulfilling the required constraints; 
iv. Generate $d_{i}$, for $i=1, \ldots, k$ fulfilling the required constraints;

v. Determine $v\left(x^{(p)}\right)$, for $p=1, \ldots, m$ using equation $(3.2)$;

vi. Calculate and store the ranking of alternatives;

vii. If cont $=n_{i t r}$ stop the procedure, else set cont $:=$ cont +1 and return to step (ii);

viii. Compute the proportion of cases in which each alternative is the best one or does not lose for a difference greater than $\epsilon$, and determine which alternatives are dominated.

\subsubsection{Generation of random parameter values}

Butler et al. (1997) described a process to generate the attribute weights respecting a given ranking according to a uniform distribution. To generate $n$ ranked attribute weights it is necessary to draw $n-1$ independent random numbers from a uniform distribution on $(0,1)$ and sort these numbers. Suppose that, for DM $i$, the sorted numbers are $r_{(n-1)}^{i} \geq \ldots \geq r_{(2)}^{i} \geq r_{(1)}^{i}$. Attribute weights are then calculated as the following differences: $w_{i 1}^{\prime}=1-r_{(n-1)}^{i}, w_{i 2}^{\prime}=r_{(n-1)}^{i}-r_{(n-2)}^{i}, \ldots, w_{i n}^{\prime}=r_{(1)}^{i}-0$. Then the numbers $w_{i j}^{\prime}$ are sorted, resulting in the sorted set of numbers $\left(w_{i 1}, w_{i 2}, \ldots, w_{i n}\right)$. These numbers add up to 1 and are uniformly distributed on the unit simplex defined by the rank-order constraints (3.3). The process described by Butler et al. (1997) assumes no ties between weights. However, if the DMs are able to identify ties between some weights, it is possible to solve the problem generating random convex combinations of the vertices of the admissible region. The multipliers used in the combinations can be generated using the process described by Butler et al. (1997) (without ranking the final values). The process of finding the vertices is outlined by Kmietowicz and Pearman (1984).

Note that the original procedure as developed by Butler et al. (1997) did not take into account the ranking condition and produces un-sorted weight vectors. However, it is easy to show that sorting does not destroy the property of a uniform distribution. This can best be shown graphically as illustrated in Figure 1. Consider a case of three attributes, and assume that weights must fulfill the condition $w_{1} \geq w_{2} \geq w_{3}$. In Figure 1, all sets of weights that satisfy $\sum_{j=1}^{3} w_{j}=1$ correspond to convex combinations of the three corner points of the triangle, and thus to points inside the triangle. The shaded region labelled "I" then represents those weight vectors that fulfill the given ranking. The process would generate points which are uniformly distributed across the entire triangle. Whenever a sorting procedure exchanges two values, a point is reflected along one of the lines splitting the large triangle, until it ends up in the region marked "I". All of these transformations leave distances between points originating in the same triangle unchanged, so points which are uniformly distributed in any of the other sub-triangles will also be uniformly distributed when mapped into sub-triangle "I".

To generate the DMs' weights $\left(d_{i}, i=1, \ldots, k\right)$, we draw $k-1$ independent random numbers from a uniform distribution on $(0,1)$ and sort these numbers. Given the sorted numbers $r_{(k-1)} \geq \ldots \geq r_{(2)} \geq r_{(1)}$, the DMs' importance weights are given by the following differences: $d_{1}=1-r_{(k-1)}, d_{2}=r_{(k-1)}-r_{(k-2)}$, $\ldots, d_{k}=r_{(1)}-0$. In the case a dictator is not allowed, as defined previously, then we discard all obtained vectors containing a highest weight greater than 0.5 .

If the DMs provide ordinal information about the value of each alternative in each attribute, for DM $i$, the attribute values $s_{i j}^{p}$ can be generated from a uniform distribution in the interval $(0,1)$ and then normalized attribute-wise in such a way that the highest value in each attribute is one and the lowest value is zero. These values can then be sorted and assigned to possible outcome values in the order indicated by the DM.

If the DMs provide ordinal information about the value of each alternative in each attribute and ordinal information about the differences of values between consecutive alternatives in each attribute, the procedure proposed by Butler et al. (1997), can be adopted to generate the required value vectors. First, the procedure is applied to generate vectors of differences $\Delta_{i j}^{l}(i=1, \ldots, k, j=1, \ldots, n$ and $l=1, \ldots, m-1)$. Note that these differences sum up to one, this property is maintained by sorting them. The sorted differences are then arranged in the ordering required by the DM. Assume, without loss of generality, that the differences are already sorted, so that $\Delta_{i k}^{1}>\Delta_{i j}^{2}>\ldots$, and indicate the rank that the DM has assigned to the value difference between $s_{i j}^{l}$ and $s_{i j}^{l+1}$ by $r_{i}$. Furthermore, let the values be arranged in decreasing order of preference. The values are then generated starting from one end of the scale by setting $s_{i j}^{1}=1$ and then recursively as $s_{i j}^{l+1}=s_{i j}^{l}-\Delta_{i j}^{l}$. 


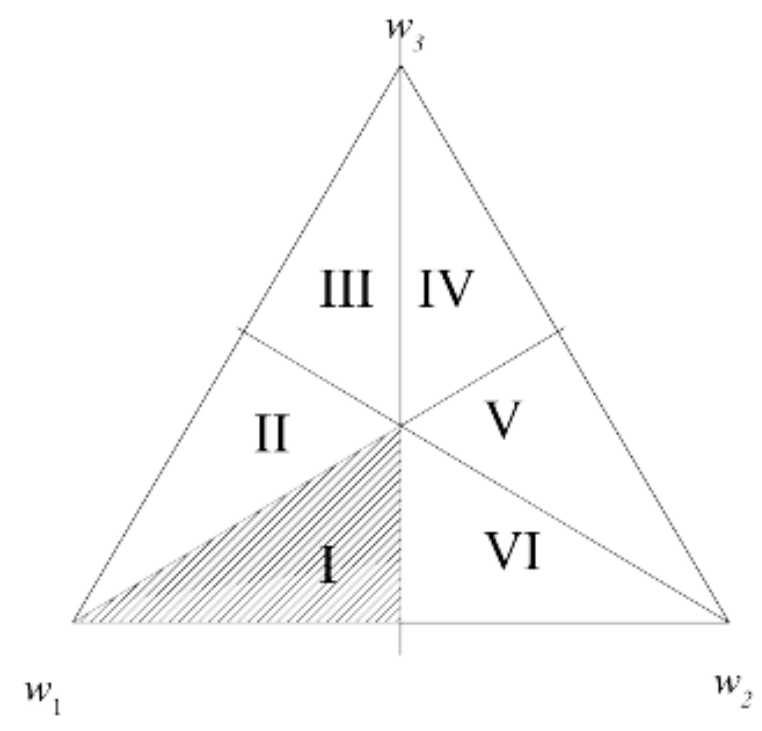

Figure 1: Generating uniformly distributed sorted weight vectors

\section{Illustrative example}

\subsection{Introduction}

In this section we illustrate the approach described in Section 3 with an example. The problem refers to an hypothetical situation in which a company wants to organize a three days workshop about its future strategy in a district capital of Portugal. The alternative locations are to be evaluated on 5 attributes: conditions of accommodation and meeting rooms, convenience of transportation for company officials, convenience of transportation for invited guests from abroad, availability of cultural activities, and prospects for outdoor activities. There are 20 alternatives $\left(x^{(1)}\right.$ : Aveiro, $x^{(2)}$ : Beja, $x^{(3)}$ : Braga, $x^{(4)}$ : Bragança, $x^{(5)}$ : Castelo Branco, $x^{(6)}$ : Coimbra, $x^{(7)}$ : Évora, $x^{(8)}$ : Faro, $x^{(9)}$ : Funchal, $x^{(10)}$ : Guarda, $x^{(11)}$ : Leiria, $x^{(12)}$ : Lisboa, $x^{(13)}$ : Ponta Delgada, $x^{(14)}$ : Portalegre, $x^{(15)}$ : Porto, $x^{(16)}$ : Santarém, $x^{(17)}$ : Setúbal, $x^{(18)}$ : Viana de Castelo, $x^{(19)}$ : Vila Real and $x^{(20)}$ : Viseu).

The problem was posed as a class room exercise in a $\mathrm{PhD}$ course on Group Decision Making and Negotiations. Five PhD students (the DMs) provided evaluations of the alternatives on each attribute, using a 0-20 scale, and provided attribute weights with reference to these scales. We normalized the evaluation matrices to the zero - one interval for each attribute and each DM. Attribute weights were then adjusted to compensate for the change in attribute ranges caused by this transformation.

In the next subsections, we will present the information about optimality, quasi-optimality, and dominance that can be obtained using the Monte-Carlo approach. The DMs' importance weights were not elicited, hence we will proceed using (3.7) to let these parameters vary freely (allowing dictatorial weights), or using (3.8) to restrict these weights to avoid that a DM becomes a dictator.

Concerning attribute weights and alternatives' values we consider four situations:

i. Exact information on weights and values;

ii. Exact information on values, ranking of weights;

iii. Rankings of weights and values;

iv. Ranking of the weights and ranking of the differences of values between consecutive alternatives in each attribute.

All rankings were deduced from the exact values provided by the $5 \mathrm{DMs}$, incorporating information about ties. 
The following subsections will illustrate the four cases. They are based on simulations using $n_{n i t r}=$ 5, 000 randomly generated parameter vectors, which fulfill the constraints implied by the information available. To illustrate the quasi-optimality results, we use a tolerance $\epsilon$ equal to $0.03,0.05$ and 0.1 . This number of generated vectors and these tolerance values are also used, for example, by Sarabando and Dias (Sarabando and Dias, 2009). It is less than the number of 10,000 vectors which is sometimes recommended in SMAA (Tervonen and Lahdelma, 2007). However, it still provides a relative accuracy of $1.4 \%$ (as compared to $1.0 \%$ for 10,000 vectors), and given that this example only serves to illustrate our approach, we consider that accuracy to be sufficient for the purpose of illustration. The simulations were run on the @Risk (TM) simulation software (http://www.palisade.com/risk) using the default random number generator settings. Running times were less than one minute for each simulation setting using an Intel Core i5 2.30 GHz processor computer with 8GB of RAM.

\subsection{Attribute weights and values are given (DMs' weights not elicited)}

In this subsection, we consider that all information is complete except for the importance of the DMs. This situation will provide reference results for comparison with situations in which the DMs provide only incomplete information.

The proportion of cases in which each alternative is the best one (optimal) or does not lose for a significant difference (quasi-optimal) is depicted in Table 1. The necessary preference relation is depicted in Table 2. Only alternatives $x^{(6)}, x^{(12)}$ and $x^{(15)}$ have positive probability of being the best ones $\left(x^{(6)}\right.$ and $x^{(12)}$, if no dictators are allowed). Alternative $x^{(12)}$ is the one with the highest probability (equal to $91.94 \%$ or $96.40 \%$, depending on whether a DM can be a dictator or not). If we consider a tolerance value equal to 0.1 , alternatives $x^{(8)}$ and $x^{(13)}\left(x^{(8)}\right.$, if no dictators are allowed) also have positive, although low, probabilities.

The example also shows the usefulness of quasi-optimality. Alternative $x^{(15)}$ has very low probability of being the best one $(0.12 \%$ or $0 \%)$ but the probability of losing for a difference greater than 0.1 is very small, i.e., in $94.40 \%\left(99.90 \%\right.$, respectively) of the cases, alternative $x^{(15)}$ does not lose for a difference greater than 0.1 . With a tolerance value equal to 0.05 , this alternative has the second highest probability. Alternative $x^{(12)}$ dominates almost all the other alternatives. Only alternatives $x^{(6)}$ and $x^{(12)}$ are not dominated by any other alternative (and $x^{(15)}$ is also not dominated if no dictators are allowed).

In this part of our example, the set of alternatives which have a positive probability of being optimal is the same as the set of alternatives which are not dominated by any other alternative. This is not always the case. A positive probability of being optimal implies that the alternative cannot be dominated, since dominance would require that the dominating alternative is better for all parameter vectors, and then the dominated alternative can never be the best one. The converse, however, is not true. It is possible that different alternatives are better than a given alternative for different parameter vectors (and are worse for some other parameter vectors). Thus it is possible that an alternative is not dominated, but never is the best one. Here we still consider exact preference information (and only unknown DM weights), and the DMs are quite homogenous since they are all $\mathrm{PhD}$ students who are familiar with the alternatives. In our example, it is therefore quite likely that if one alternative is better than another one for some parameter (DM weight) vector, this will also hold for the other parameter vectors and therefore dominance is established.

Table 1: Proportion of cases (\%) in which each alternative is the best one $(\epsilon=0)$ or does not lose for a difference greater than $\epsilon(\epsilon=0.03,0.05,0.1)$ : exact attribute weights and values known.

\begin{tabular}{|l|c|c|c|c|c|c|c|c|}
\cline { 2 - 9 } \multicolumn{1}{c|}{} & \multicolumn{3}{c|}{ DMs' weights are free to vary } & \multicolumn{3}{c|}{ No dictators allowed (weights $\leq 0.5$ ) } \\
\hline Alt. $\backslash$ Tol. & $\epsilon=0$ & $\epsilon=0.03$ & $\epsilon=0.05$ & $\epsilon=0.1$ & $\epsilon=0$ & $\epsilon=0.03$ & $\epsilon=0.05$ & $\epsilon=0.1$ \\
\hline 6 (Coimbra) & 7.94 & 14.59 & 20.06 & 37.95 & 3.60 & 8.34 & 13.23 & 36.34 \\
8 (Faro) & 0 & 0 & 0 & 7.58 & 0 & 0 & 0 & 6.95 \\
12 (Lisboa) & 91.94 & 96.47 & 98.25 & 99.92 & 96.40 & 99.04 & 99.72 & 100 \\
13 (P. Delgada) & 0 & 0 & 0 & 0.10 & 0 & 0 & 0 & 0 \\
15 (Porto) & 0.12 & 7.45 & 24.73 & 94.40 & 0 & 3.74 & 24.12 & 99.90 \\
\hline
\end{tabular}

\subsection{Rankings of the attribute weights and precise values are given}

We now assume that each DM provided a ranking of the attribute weights and precise values. 
Table 2: Necessary preference relation for known attribute weights and values $\left(\bullet: x^{(\text {row })}\right.$ is necessarily better than $x^{(\mathrm{col})}$ given free DMs' weights; o: $x^{(\mathrm{row})}$ is necessarily better than $x^{(\mathrm{col})}$ only if no DM can be a dictator).

\begin{tabular}{|c|c|c|c|c|c|c|c|c|c|c|c|c|c|c|c|c|c|c|}
\hline & 1 & 23 & 4 & 5 & 6 & 78 & 9 & 10 & 11 & 12 & 13 & 14 & 15 & 16 & 17 & 18 & 19 & 20 \\
\hline 1 (Aveiro) & & $\bullet$ & $\bullet$ & $\bullet$ & & $\bullet$ & & $\bullet$ & $\bullet$ & & & $\bullet$ & & $\bullet$ & $\bullet$ & $\bullet$ & $\bullet$ & $\bullet$ \\
\hline 2 (Beja) & & & $\bullet$ & $\bullet$ & & & & $\bullet$ & & & & $\bullet$ & & & & & & \\
\hline 3 (Braga) & & & $\bullet$ & $\bullet$ & & & & $\bullet$ & $\bullet$ & & & $\bullet$ & & & & o & $\bullet$ & \\
\hline 4 (Bragança) & & & & & & & & & & & & & & & & & & \\
\hline 5 (C. Branco) & & & & & & & & & & & & & & & & & & \\
\hline 6 (Coimbra) & 0 & $\bullet$ & $\bullet$ & $\bullet$ & & - & & $\bullet$ & $\bullet$ & & $\bullet$ & $\bullet$ & & $\bullet$ & $\bullet$ & $\bullet$ & $\bullet$ & $\bullet$ \\
\hline 7 (Évora) & & & - & - & & & & $\bullet$ & & & & - & & & & & $\bullet$ & \\
\hline 8 (Faro) & $\bullet$ & $\bullet \bullet$ & - & $\bullet$ & & - & $\bullet$ & $\bullet$ & $\bullet$ & & $\bullet$ & $\bullet$ & & $\bullet$ & $\bullet$ & $\bullet$ & $\bullet$ & $\bullet$ \\
\hline 9 (Funchal) & & $\bullet$ & - & $\bullet$ & & & & $\bullet$ & $\bullet$ & & & - & & $\bullet$ & o & $\bullet$ & $\bullet$ & $\bullet$ \\
\hline 10 (Guarda) & & & & & & & & & & & & & & & & & & \\
\hline 11 (Leiria) & & 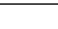 & $\bullet$ & $\bullet$ & & & & $\bullet$ & & & & $\bullet$ & & & & & $\bullet$ & \\
\hline 12 (Lisboa) & $\bullet$ & $\bullet \bullet$ & $\bullet$ & $\bullet$ & & $\bullet \bullet$ & $\bullet$ & $\bullet$ & $\bullet$ & & $\bullet$ & $\bullet$ & o & $\bullet$ & $\bullet$ & $\bullet$ & $\bullet$ & $\bullet$ \\
\hline 13 (P. Delgada) & & & $\bullet$ & $\bullet$ & & & & $\bullet$ & & & & $\bullet$ & & & & & $\bullet$ & \\
\hline 14 (Portalegre) & & & & & & & & & & & & & & & & & & \\
\hline 15 (Porto) & $\bullet$ & $\bullet \bullet$ & $\bullet$ & $\bullet$ & & $\bullet \bullet$ & $\bullet$ & $\bullet$ & $\bullet$ & & $\bullet$ & $\bullet$ & & $\bullet$ & $\bullet$ & $\bullet$ & $\bullet$ & $\bullet$ \\
\hline 16 (Santarém) & & & - & $\bullet$ & & & & $\bullet$ & & & & - & & & & & $\bullet$ & \\
\hline 17 (Setúbal) & & & - & $\bullet$ & & & & $\bullet$ & & & & - & & & & & o & \\
\hline 18 (V. Castelo) & & & $\bullet$ & $\bullet$ & & & & $\bullet$ & & & & o & & & & & $\bullet$ & \\
\hline 19 (Vila Real) & & & & $\bullet$ & & & & $\bullet$ & & & & & & & & & & \\
\hline 20 (Viseu) & & & 0 & $\bullet$ & & & & $\bullet$ & & & & $\bullet$ & & & & & $\bullet$ & \\
\hline
\end{tabular}

Table 3 shows the proportion of cases in which each alternative is the best one and also the proportion of cases in which each alternative is almost better than all other alternatives when a tolerance $\epsilon>0$ is taken into account. Table 4 represents the necessary preference relation for this setting. As expected, since there is a loss of information when working with rankings rather than precise weights, this relation contains fewer elements than before.

Since the results obtained considering no DM can be a dictator were not much different from the ones obtained generating the DMs' weights considering (3.7), we will refer henceforth to the latter. Once again, alternative $x^{(12)}$ has the highest probability of being the best one. Alternative $x^{(8)}$ appears with positive probability of being the best one (despite this probability being very low). Only alternatives $x^{(6)}, x^{(8)}, x^{(12)}$ and $x^{(15)}$ are not dominated by any other alternative.

Table 3: Proportion of cases (\%) in which each alternative is the best one $(\epsilon=0)$ or does not lose for a difference greater than $\epsilon(\epsilon=0.03,0.05,0.1)$ : ranking of the attribute weights and exact values known.

\begin{tabular}{|l|c|c|c|c|c|c|c|c|}
\cline { 2 - 9 } \multicolumn{1}{c|}{} & \multicolumn{4}{c|}{ DMs' weights are free to vary } & \multicolumn{3}{c|}{ No dictators allowed (weights $\leq 0.5$ ) } \\
\hline Alt. $\backslash$ Tol. & $\epsilon=0$ & $\epsilon=0.03$ & $\epsilon=0.05$ & $\epsilon=0.1$ & $\epsilon=0$ & $\epsilon=0.03$ & $\epsilon=0.05$ & $\epsilon=0.1$ \\
\hline 6 (Coimbra) & 4.00 & 7.63 & 11.02 & 23.77 & 1.43 & 3.92 & 7.06 & 19.10 \\
8 (Faro) & 0.01 & 0.18 & 0.79 & 11.22 & 0 & 0.14 & 0.50 & 9.41 \\
9 (Funchal) & 0 & 0 & 0 & $<0.01$ & 0 & 0 & 0 & 0 \\
12 (Lisboa) & 95.31 & 97.98 & 98.85 & 99.77 & 98.53 & 99.54 & 99.84 & 99.98 \\
13 (P. Delgada) & 0 & 0 & $<0.01$ & 0.19 & 0 & 0 & 0 & 0 \\
15 (Porto) & 0.68 & 7.41 & 25.70 & 94.91 & 0.04 & 4.88 & 24.90 & 98.19 \\
\hline
\end{tabular}

\subsection{Rankings of the attribute weights and rankings of the values are given}

We now further weaken the information and suppose that the DMs provide rankings of the alternatives' values on each attribute, possibly with ties (rather than precise values), as well as rankings of the attribute weights. We will consider the rankings that correspond to the exact values used in the previous subsections.

Tables 5 and 6 represent the results for this case similarly to the previous subsections. Considering a tolerance equal to 0.1 , there are 9 alternatives with positive probability ( 5 of them with very low probabilities). Alternatives $x^{(6)}, x^{(8)}, x^{(12)}, x^{(13)}$ and $x^{(15)}$ are the only ones to which no other alternative is necessarily preferred, this does not longer hold for $x^{(13)}$ if we exclude dictatorial weights. Here we also see an example of an alternative which is not dominated, but still has zero probability of being optimal, this happens for $x^{(13)}$ and free weights. 
Table 4: Necessary preference relation given rankings of the attribute weights and exact values known •: free DMs' weights; ○: weights $<0.5$ (no dictator).

\begin{tabular}{|c|c|c|c|c|c|c|c|c|c|c|c|c|c|c|c|c|c|c|}
\hline & 1 & $\begin{array}{ll}2 & 3\end{array}$ & 4 & 5 & 67 & 78 & 9 & 10 & 11 & 12 & 13 & 14 & 15 & 16 & 17 & 18 & 19 & 20 \\
\hline 1 (Aveiro) & & 0 & -• & - & & & & 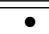 & & & & $\begin{array}{l}\bullet \\
\end{array}$ & & 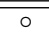 & o & o & $\begin{array}{l}\bullet \\
\end{array}$ & \\
\hline 2 (Beja) & & & $\bullet$ & $\bullet$ & & & & $\bullet$ & & & & $\bullet$ & & & & & o & \\
\hline 3 (Braga) & & & $\bullet$ & $\bullet$ & & & & $\bullet$ & $\bullet$ & & & 0 & & & & & o & \\
\hline 4 (Bragança) & & & & & & & & & & & & & & & & & & \\
\hline 5 (C. Branco) & & & & & & & & & & & & & & & & & & \\
\hline 6 (Coimbra) & 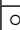 & $\bullet$ & $\bullet$ & $\bullet$ & 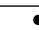 & $\bullet$ & & $\bullet$ & $\bullet$ & & $\bullet$ & $\bullet$ & & $\bullet$ & $\bullet$ & $\bullet$ & $\bullet$ & $\bullet$ \\
\hline 7 (Évora) & & & $\bullet$ & $\bullet$ & & & & $\bullet$ & & & & $\bullet$ & & $\bullet$ & & & 0 & \\
\hline 8 (Faro) & 0 & $\bullet$ & $\bullet$ & $\bullet$ & 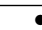 & - & o & $\bullet$ & $\bullet$ & & & $\bullet$ & & $\bullet$ & $\bullet$ & $\bullet$ & $\bullet$ & $\bullet$ \\
\hline 9 (Funchal) & & & $\bullet$ & $\bullet$ & & & & $\bullet$ & & & & $\bullet$ & & & & 0 & $\bullet$ & \\
\hline 10 (Guarda) & & & & & & & & & & & & & & & & & & \\
\hline 11 (Leiria) & & & $\bullet$ & - & & & & - & & & & 0 & & & & & 0 & \\
\hline 12 (Lisboa) & $\bullet$ & $\bullet \bullet$ & $\bullet$ & $\bullet$ & 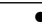 & - 0 & $\bullet$ & $\bullet$ & $\bullet$ & & 0 & $\bullet$ & & $\bullet$ & $\bullet$ & $\bullet$ & $\bullet$ & $\bullet$ \\
\hline 13 (P. Delgada) & & & $\bullet$ & $\bullet$ & & & & $\bullet$ & & & & & & & & & 0 & \\
\hline 14 (Portalegre) & & & & & & & & & & & & & & & & & & \\
\hline 15 (Porto) & $\bullet$ & $\bullet \bullet$ & $\bullet$ & $\bullet$ & & & & $\bullet$ & $\bullet$ & & 0 & $\bullet$ & & $\bullet$ & $\bullet$ & $\bullet$ & $\bullet$ & $\bullet$ \\
\hline 16 (Santarém) & & & $\bullet$ & $\bullet$ & & & & $\bullet$ & & & & $\bullet$ & & & & & 0 & \\
\hline 17 (Setúbal) & & & $\bullet$ & $\bullet$ & & & & $\bullet$ & & & & $\bullet$ & & & & & 0 & \\
\hline 18 (V. Castelo) & & & $\bullet$ & $\bullet$ & & & & $\bullet$ & & & & 0 & & & & & 0 & \\
\hline 19 (Vila Real) & & & & $\bullet$ & & & & & & & & & & & & & & \\
\hline 20 (Viseu) & & & 0 & $\bullet$ & & & & $\bullet$ & & & & $\bullet$ & & & & & $\bullet$ & \\
\hline
\end{tabular}

Table 5: Proportion of cases (\%) in which each alternative is the best one $(\epsilon=0)$ or does not lose for a difference greater than $\epsilon(\epsilon=0.03,0.05,0.1)$ : ranking of the attribute weights and ranking of the values.

\begin{tabular}{|l|c|c|c|c|c|c|c|c|}
\cline { 2 - 9 } \multicolumn{1}{c|}{} & \multicolumn{3}{c|}{ DMs' weights are free to vary } & \multicolumn{3}{c|}{ No dictators allowed (weights $\leq 0.5$ ) } \\
\hline Alt. $\backslash$ Tol. & $\epsilon=0$ & $\epsilon=0.03$ & $\epsilon=0.05$ & $\epsilon=0.1$ & $\epsilon=0$ & $\epsilon=0.03$ & $\epsilon=0.05$ & $\epsilon=0.1$ \\
\hline 1 (Aveiro) & 0 & 0 & 0.01 & 0.23 & 0 & 0 & 0 & 0.10 \\
3 (Braga) & 0 & 0 & 0 & $<0.01$ & 0 & 0 & 0 & 0 \\
6 (Coimbra) & 1.95 & 5.03 & 8.73 & 25.79 & 0.64 & 2.51 & 5.15 & 22.07 \\
7 (Évora) & 0 & 0 & 0 & 0.01 & 0 & 0 & 0 & 0 \\
8 (Faro) & 0.12 & 0.99 & 3.46 & 31.73 & 0.06 & 0.64 & 2.97 & 31.68 \\
9 (Funchal) & 0 & $<0.01$ & $<0.01$ & 0.09 & 0 & 0 & 0 & 0.04 \\
12 (Lisboa) & 96.28 & 99.26 & 99.69 & 99.95 & 98.56 & 99.76 & 99.96 & 99.98 \\
13 (P. Delgada) & 0 & 0 & $<0.01$ & 0.08 & 0 & 0 & 0 & 0 \\
15 (Porto) & 1.65 & 38.80 & 74.38 & 98.56 & 0.74 & 37.37 & 75.56 & 99.34 \\
\hline
\end{tabular}

Table 6: Necessary preference relations given rankings of the attribute weights and rankings of the values •: free DMs' weights; ०: weights $<0.5$ (no dictator).

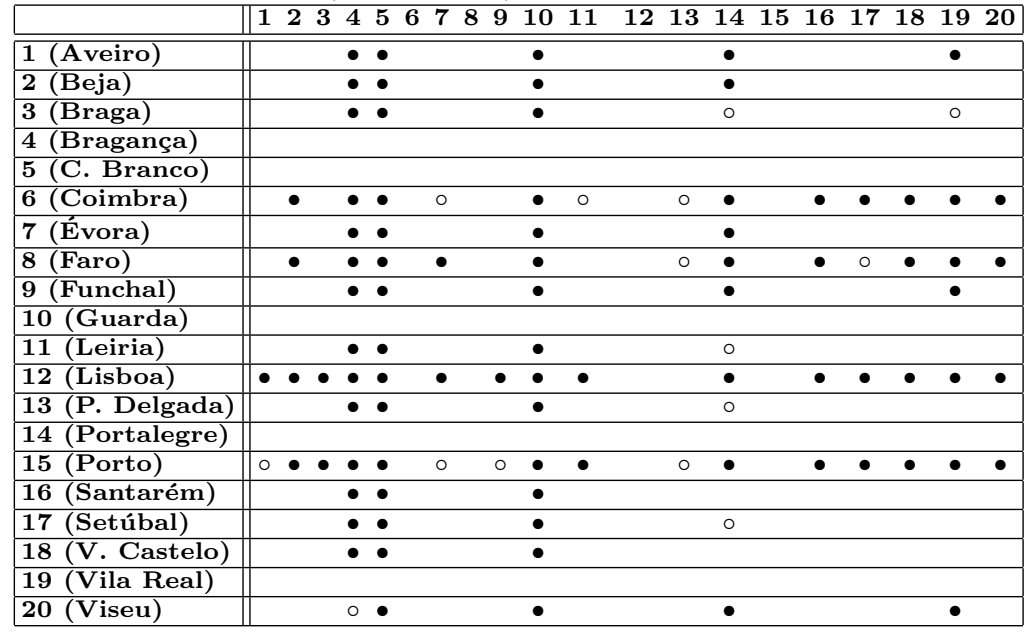




\subsection{Rankings of the attribute weights and rankings of the differences of values between consecutive alternatives in each attribute are given}

We now consider an intermediate situation concerning information about values: rather than eliciting precise values or a simple ranking of these values, we assume that the DMs provide the ranking of the values plus a ranking of differences of values between consecutive alternatives in each attribute.

The results for this case are shown in Tables 7 and 8 . The results are not very different to the ones obtained previously. Nevertheless, it should be noted that the results presented in Table 7 (ranking of attribute weights and ranking of the differences of values between consecutive alternatives in each attribute) are closer to the results presented in Table 1 (attribute weights and values known) than to the results in Table 5 (ranking of the attribute weights and ranking of the values). Alternatives $x^{(6)}, x^{(8)}$, $x^{(12)}$ and $x^{(15)}$ are the only ones to which no other alternative is necessarily preferred.

Table 7: Proportion of cases (\%) in which each alternative is the best one $(\epsilon=0)$ or does not lose for a difference greater than $\epsilon(\epsilon=0.03,0.05,0.1)$ : ranking of the attribute weights and ranking of the differences of values between consecutive alternatives in each attribute.

\begin{tabular}{|l|c|c|c|c|c|c|c|c|}
\cline { 2 - 9 } \multicolumn{1}{c|}{} & \multicolumn{3}{c|}{ DMs' weights are free to vary } & \multicolumn{3}{c|}{ No dictators allowed (weights $\leq 0.5$ ) } \\
\hline Alt.\Tol. & $\epsilon=0$ & $\epsilon=0.03$ & $\epsilon=0.05$ & $\epsilon=0.1$ & $\epsilon=0$ & $\epsilon=0.03$ & $\epsilon=0.05$ & $\epsilon=0.1$ \\
\hline 1 (Aveiro) & 0 & 0 & 0 & $<0.01$ & 0 & 0 & 0 & 0 \\
6 (Coimbra) & 9.50 & 14.00 & 17.74 & 30.33 & 6.15 & 10.11 & 13.51 & 26.47 \\
8 (Faro) & 0.01 & 0.11 & 0.57 & 8.71 & 0 & 0.12 & 0.44 & 7.52 \\
9 (Funchal) & 0 & 0 & 0 & 0.01 & 0 & 0 & 0 & 0.02 \\
12 (Lisboa) & 90.05 & 93.60 & 95.14 & 97.58 & 93.77 & 96.36 & 97.53 & 99.20 \\
13 (P. Delgada) & 0 & 0.01 & 0.03 & 0.60 & 0 & 0 & 0 & 0.06 \\
15 (Porto) & 0.45 & 5.56 & 21.47 & 84.43 & 0.08 & 4.02 & 27.78 & 88.64 \\
\hline
\end{tabular}

Table 8: Necessary preference relation given rankings of the attribute weights and rankings of the differences of values between consecutive alternatives in each attribute $\bullet$ : free DMs' weights; ०: weights $<0.5$ (no dictator).

\begin{tabular}{|c|c|c|c|c|c|c|c|c|c|c|c|c|c|c|c|c|c|}
\hline & $\mid 1$ & 23 & 34 & 5 & 56 & 78 & 89 & 10 & 11 & 12 & 13 & 1415 & 16 & 17 & 18 & 19 & 20 \\
\hline 1 (Aveiro) & & o & $\bullet$ & $\bullet$ & & & & $\bullet$ & o & & & $\bullet$ & ○ & ○ & $\bullet$ & $\bullet$ & \\
\hline 2 (Beja) & & & • & • & & & & $\bullet$ & & & & $\bullet$ & & & & 0 & \\
\hline 3 (Braga) & & & • & - & & & & $\bullet$ & $\bullet$ & & & 0 & & & $\bullet$ & 0 & \\
\hline \multicolumn{18}{|l|}{4 (Bragança) } \\
\hline \multicolumn{18}{|l|}{5 (C. Branco) } \\
\hline 6 (Coimbra) & 0 & $\bullet$ & $\bullet$ & $\bullet$ & & $\bullet$ & & $\bullet$ & $\bullet$ & & $\bullet$ & $\bullet$ & $\bullet$ & $\bullet$ & $\bullet$ & $\bullet$ & $\bullet$ \\
\hline 7 (Évora) & & & • & ? & & & & $\bullet$ & & & & $\bullet$ & & & o & 0 & \\
\hline 8 (Faro) & & $\bullet$ & $\bullet$ & 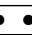 & & $\bullet$ & & $\bullet$ & $\bullet$ & & & $\bullet$ & $\bullet$ & $\bullet$ & $\bullet$ & $\bullet$ & $\bullet$ \\
\hline 9 (Funchal) & & & & $\bullet$ & & & & $\bullet$ & & & & $\bullet$ & & & $\circ$ & $\bullet$ & \\
\hline \multicolumn{18}{|l|}{10 (Guarda) } \\
\hline 11 (Leiria) & & & $\bullet$ & $\bullet$ & & & & $\bullet$ & & & & $\circ$ & & & $\bullet$ & & \\
\hline 12 (Lisboa) & $\bullet$ & $\bullet \bullet$ & $\bullet \bullet$ & $\bullet$ & & $\bullet \circ$ & ○・ & $\bullet$ & $\bullet$ & & & $\bullet$ & $\bullet$ & $\bullet$ & $\bullet$ & $\bullet$ & $\bullet$ \\
\hline 13 (P. Delgada) & & & $\bullet$ & $\bullet$ & & & & $\bullet$ & & & & & & & & $\circ$ & \\
\hline \multicolumn{18}{|l|}{14 (Portalegre) } \\
\hline 15 (Porto) & $\mid$ & $\bullet \bullet$ & - $\bullet$ & $\bullet$ & & & & $\bullet$ & $\bullet$ & & & $\bullet$ & $\bullet$ & $\bullet$ & $\bullet$ & $\bullet$ & $\bullet$ \\
\hline 16 (Santarém) & & & $\bullet$ & $\bullet$ & & & & $\bullet$ & & & & $\bullet$ & & & & & \\
\hline 17 (Setúbal) & & & • & $\bullet$ & & & & $\bullet$ & & & & & & & & & \\
\hline 18 (V. Castelo) & & & $\bullet$ & $\bullet$ & & & & $\bullet$ & & & & & & & & & \\
\hline \multicolumn{18}{|l|}{19 (Vila Real) } \\
\hline 20 (Viseu) & & & 0 & 5 & & & & $\bullet$ & & & & $\bullet$ & & & & $\bullet$ & \\
\hline
\end{tabular}

\subsection{Discussion}

In all the cases, it was possible to conclude that alternative $x^{(12)}$ (Lisboa) is clearly the best alternative, but if the DMs would like to analyze with more detail a small subset of alternatives it is possible to recommend also alternatives $x^{(6)}$ and $x^{(15)}$ (Coimbra and Porto, respectively), and possibly alternative $x^{(8)}$ (Faro). In Figure 2 it is possible to see how the optimality and quasi-optimality volume of these four alternatives increases with the used tolerance, in the four cases referred previously, and considering that DMs' weights are free to vary. Using tolerance values equal to 0.05 and 0.1 , a promising alternative appeared, $x^{(15)}$, that has very low probabilities of being the best one, but very high probability of not losing for a difference greater than 0.1. Considering the different types of incomplete information, the results are not very different. The main difference appeared in the probabilities of alternative $x^{(15)}$ 
considering a ranking of the attribute weights and a ranking of the values. Using the necessary preference relation, in all the cases, it was possible to eliminate most of the alternatives.
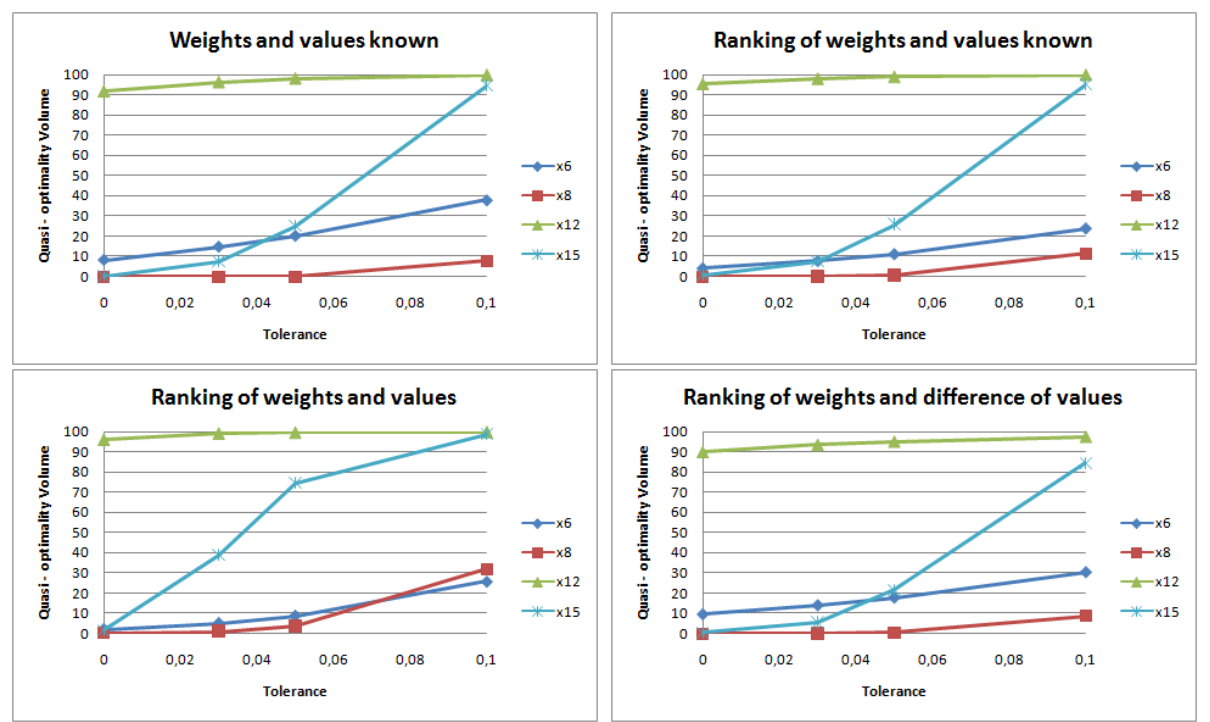

Figure 2: Optimality / quasi-optimality volume - DMs' weights are free to vary.

The main results remain valid if one considers only non-dictator weights. The main difference is that, in all the cases the proportion of cases in which alternative $x^{(12)}$ is the best one increases, decreasing the correspondent proportion for alternative $x^{(6)}$. This happens because three DMs agree that alternative $x^{(12)}$ is the best one.

In the presented example, Lisboa is clearly the best option to locate the workshop. However, what happens if the company decides that the workshop should not take place in Lisboa? We did some experiments considering that Lisboa cannot be chosen (i.e., eliminating alternative $\left.x^{(12)}\right)^{1}$. In this case, alternative $x^{(15)}$ (Porto) appeared as being the most promising alternative, because it is the one with highest probability of being the best one. Once again, only alternatives $x^{(6)}$ and $x^{(8)}$ might be considered for further analysis. Note that, when considering a tolerance of $0, x^{(15)}$ would not appear to be the best alternative to Lisboa, but its interest becomes evident when a tolerance of 0.05 or 0.1 is considered.

\section{Conclusions}

In this work we addressed the problem in which a group of DMs is not able to, or does not want to, provide complete information about their individual preferences. We considered ordinal information in the form of a ranking of the attribute weights, a ranking of the value of each alternative under each attribute and a ranking of the differences of values between consecutive alternatives in each attribute. By exploring the parameter space (DMs' weights, attribute weights, and values) we can check if there are optimal (or quasi-optimal) alternatives for a wide range of parameter values, and check if there are alternatives that are necessarily or with high probability preferred to the other ones, allowing discarding the latter.

Our approach does not require DMs to define the DMs' importance weights. Thereby we avoid the problem of assessing importance weights, which is one of the difficulties associated with the use of additive group value functions. We also proposed constraining the set of DMs' weights in a way that would prevent a single DM from becoming a dictator, in the sense of Sarabando and Dias (Dias and Sarabando, 2012), which seems to suit this context better than Arrows's classical definition.

\footnotetext{
${ }^{1}$ When removing Lisbon we recomputed everything from the start and in particular renormalize the scales. This is, we normalized the value matrices and recalculated the weights in order to neutralize the effect of the normalization of the value of the alternatives.
} 
Monte-Carlo simulation was used to approximate the relative volume of the parameter space domain where each condition holds, with respect to the domain of the feasible parameter values. Assuming a uniform distribution, this relative volume corresponds to the probability that the condition under study holds. Alternatives with very low (or zero) probability of being optimal might be discarded. The approach also enables to identify alternatives which dominate the other ones, without solving complex optimization problems. The use of simulation is more general and flexible than the use of optimization models, although it does not yield perfectly accurate results. The number of iterations considered in the simulations can be as high as necessary to provide the desired accuracy. Furthermore, it is possible to combine simulation and optimization methods (Kadzinski and Tervonen, 2013) to obtain the benefits of both methods.

The presented approach can be easily used and can be very useful to recommend a small subset of alternatives to the DMs. This reduced set of alternatives can be subject to a more exhaustive analysis by the DMs. The illustrative example demonstrates how this methodology can be used, and what kind of information it can provide to the DMs.

In future work, a more exhaustive study to test the approach should be done. It is important to test, for example, if the results obtained from a ranking of values and a ranking of differences are significantly different. This would allow to evaluate the trade-off between quality of results on the one hand, and the additional effort required from DMs to specify differences instead of just ranks on the other hand. A first step in that direction was taken in Vetschera et al (2014).

Rankings of values, and rankings of differences are of course only two points on a continuum of different levels of information the DMs might provide. We considered the case of complete rankings, but the rankings provided could also be incomplete, if the comparison between some alternatives turns out to be too difficult for a DM. On the other hand, in the present paper, information on single-attribute value functions and on weights was provided separately, but it would be possible to deal with holistic comparison of alternatives concerning all attributes simultaneously.

The approach proposed here is not limited to the additive group value function. Using simulation, other, more complex forms of aggregation can also be studied. This includes, for instance, the Choquet integral which has been addressed by Angilella et al. (2012) for the single DM case. Of course, extension of our approach in these directions is not only a technical problem of developing different types of simulations, but also involves conceptual problems with respect to the axiomatic basis of such alternative group aggregation procedures.

\section{References}

Angilella, S., Corrente, S., Greco, S., 2012. SMAA-Choquet: Stochastic Multicriteria Acceptability Analysis for the Choquet Integral. Advances in Computational Intelligence 300, 248-257.

Alfares, H., Duffuaa, S., 2008. Assigning cardinal weights in multi-criteria decision making based on ordinal ranking. Journal of Multi-Criteria Decision Analysis 15(5/6), 125-133.

Arrow, K., 1951. Social Choice and Individual Values. John Wiley and Sons, New York.

Bana e Costa, C., 1986. A multicriteria decision aid methodology to deal with conflicting situations on the weights. European Journal of Operational Research 26, 22-34.

Belton, V., Stewart, T., 2002. Multiple Criteria Decision Analysis: An Integrated Approach. Kluwer Academic, Dordrecht.

Butler, J., Jia, J., Dyer, J., 1997. Simulation techniques for the sensitivity analysis of multi-criteria decision models. European Journal of Operational Research 103(3), 531-546.

Charnetski, J., Soland, R., 1978. Multiple-attribute decision making with partial information: The comparative hypervolume criterion. Naval Research Logistics Quarterly 25, 279-288.

Contreras, I., Marmol, A., 2007. A lexicographical compromise method for multiple criteria group decision problems with imprecise information. European Journal of Operational Research 181(3), 1530-1539.

Cook, W., Kress, M., 1985. Ordinal ranking with intensity of preference. Management Science 31,26-32. 
Damart, S., Dias, L., Mousseau, V., 2007. Supporting groups in sorting decisions: Methodology and use of a multi-criteria aggregation / disaggregation DSS. Decision Support Systems 43(4), 1464-1475.

Dias, L., Clímaco, J., 2000. Additive aggregation with variable interdependent parameters: The VIP analysis software. Journal of the Operational Research Society 51(9), 1070-1082.

Dias, L., Clímaco, J., 2005. Dealing with imprecise information in group multicriteria decisions: A methodology and a GDSS architecture. European Journal of Operational Research 160, 291-307.

Dias, L., Sarabando, P., 2012. A note on a group preference axiomatization with cardinal utility. Decision Analysis 9(3), 231-237.

Dyer, J., Sarin, R., 1979. Group preference aggregation rules based on strength of preference. Management Science 25(9), 822-832.

Edwards, W., Barron, F., 1994. SMART and SMARTER: Improved simple methods for multiattribute utility measurement. Organizational Behavior and Human Decision Processes 60, 306-325.

Eklund, P., Rusinowska, A., De Swart, H., 2007. Consensus reaching in committees, European Journal of Operational Research 178, 185-193.

Figueira, J., Greco, S., Ehrgott, M., 2005. Multiple Criteria Decision Analysis: State of the Art Surveys. Springer.

Giarlotta, A., Greco, S., 2013. Necessary and possible preference structures. Journal of Mathematical Economics 49(2), 163-172.

Greco, S., Mousseau, M., Slowinski, R., 2008. Ordinal regression revisited: multiple criteria ranking using a set of additive value functions. European Journal of Operational Research 191(2), 415-435.

Hazen, G., 1986. Partial information, dominance, and potential optimality in multiattribute utility theory. Operations Research 34(2), 296-310.

Hodgkin, J., Belton, V., Koulouri, A., 2005. Supporting the intelligent MCDA user: A case study in multi-person multi-criteria decision support. European Journal of Operational Research 160, 172-189.

Hwang, C., Lim, M., 1987, Group Decision Making under Multiple Criteria - Methods and Applications Springer, Berlin.

Jabeur, K., Martel, J.-M., 2007. A collective choice method based on individual preferences relational systems (p.r.s.). European Journal of Operational Research 177, 1549-1565.

Jiménez, A., Mateos, A., Ríos-Insua, S., 2005. Monte Carlo simulation techniques in a decision support system for group decision making. Group Decision and Negotiation 14, 109-130.

Kadzinski, M., Salvatore, G., Slowinski, R. 2013. Robust multi-criteria ranking with additive value models and holistic pair-wise preference statements. European Journal of Operational Research 228(1), 169180.

Kadzinski, M., Tervonen, T., 2013. Robust multi-criteria ranking with additive value models and holistic pair-wise preference statements. European Journal of Operational Research 228(1), 169-180.

Keeney, R., 1976. A group preference axiomatization with cardinal utility. Management Science 23(2), 140-145.

Keeney, R. L., 2013. Foundations for Group Decision Analysis. Decision Analysis 10(2), 103-120.

Keeney, R., Kirkwood, C., 1975. Group decision making using cardinal social welfare functions. Management Science 22(4), 430-437.

Keeney, R. L., Nau, R., 2011. A theorem for Bayesian group decisions. Journal of Risk and Uncertainty $43,1-17$. 
Keeney, R., Raiffa, H., 1976. Decisions with Multiple Objectives: Preferences and Value Tradeoffs. J. Wiley and Sons, New York.

Kemeny, J., Snell, J., 1962. Preference ranking: An axiomatic approach, in Mathematical Models in the Social Sciences, Blaisdell, New York, pp. 9-32.

Khélifa, S., Martel, J.-M., 2001. A distance-based collective weak ordering. Group Decision and Negotiation 10, 317-329.

Kim, S., Ahn, B., 1999. Interactive group decision making procedure under incomplete information. European Journal of Operational Research 116, 498-508.

Kirkwood, C. W., Sarin, R. K., 1985. Ranking with partial information: A method and an application. Operations Research 33(1), 38-48.

Kmietowicz, A., Pearman, A., 1984. Decision theory, linear partial information and statistical dominance. Omega 12, 391-399.

Lahdelma, R., Salminen, J., 2001. SMAA-2: Stochastic multicriteria acceptability analysis for group decision making. Operations Research 49, 444-454.

Lahdelma, R., Miettinen, K., Salminen, P., 2003. Ordinal criteria in stochastic multicriteria acceptability analysis (SMAA). European Journal of Operational Research 147(1), 117-127.

Lahdelma, R., Hokkanen, J., Salminen, P., 1998. SMAA - Stochastic multiobjective acceptability analysis. European Journal of Operational Research 106(1), 137-143.

Leskinen, P., Viitanen, J., Kangas, A., Kangas, J., 2006. Alternatives to Incorporate Uncertainty and Risk Attitude in Multicriteria Evaluation of Forest Plans, Forest science 52(3), 304-312.

Mateos, A., Jiménez, A., Ríos-Insua, S., 2003. A multiattribute solving dominance and potential optimality in imprecise multiattribute additive problems. Reliability Engineering and System Safety 79, 253-262.

Park, K. S., Kim, S. H., 1997. Tools for interactive multiattribute decision making with incompletely identified information. European Journal of Operational Research 98(1), 111-123.

Pöyhönen, M., Hämäläinen, R. P., 2001. On the convergence of multiattribute weighting methods. European Journal of Operational Research 129(3), 569-585.

Raiffa, H., 1982. Art and Science of Negotiation. Harvard University Press.

Salo, A., Hämäläinen, R., 2001. Preference ratio in multiattribute evaluation (PRIME) - elicitation and decision procedures under incomplete information. IEEE Transactions on Systems, Man and Cybernetics, Part A: Systems and Humans 31(6), 533-545.

Sarabando, P., Dias, L., 2009. Multi-attribute choice with ordinal information: A comparison of different decision rules. IEEE Transactions on Systems, Man, and Cybernetics, Part A: Systems and Humans $39(3), 545-554$.

Sarabando, P., Dias, L., Vetschera, R., 2013. Mediation with incomplete information: Approaches to suggest potential agreements. Group Decision and Negotiation 22, 561-597.

Salo, A., 1995. Interactive decision aiding for group decision support. European Journal of Operational Research 84, 134-149.

Schoemaker, P., Waid, C., 1982. An experimental comparison of different approaches to determining weights in additive utility models. Management Science 28, 182-196.

Sen, A., 1970. Collective Choice and Social Welfare. Holden-Day, San Francisco, CA.

Tavares, L., 2004. A model to support the search for consensus with conflicting rankings: Multitrident. International Transactions in Operational Research 11, 107-115. 
Tervonen, T., Lahdelma, R., 2007. Implementing stochastic multicriteria acceptability analysis. European Journal of Operational Research 178(2), 500-513.

Tervonen, T., Figueira, J., 2008. A Survey on stochastic multicriteria acceptability analysis methods. Journal of Multicriteria Decision Analysis 15, 1-14.

Vetschera, R., 1990. Group decision and negotiation support - a methodological survey. OR Spektrum 12, $67-77$.

Vetschera, R., 2009. Learning about preferences in electronic negotiations - a volume based measurement method. European Journal of Operational Research 194, 452-463.

Vetschera, R., Dias, L., Sarabando, P., 2014. Levels of incomplete information in group decision models - A comprehensive simulation study. Computers and Operations Research 51, 160-171.

Watson, S., Buede, D., 1987. Decision synthesis. The principles and practice of decision analysis. Cambridge University Press, Cambridge.

Weber, M., 1987. Decision making with incomplete information. European Journal of Operational Research 28(1), 44-57.

von Winterfeldt, D., Edwards, W., 1986. Decision Analysis and Behavioral Research. Cambridge University Press, Cambridge. 